\title{
Endovascular management of aortic coarctation in an adult patient
}

\begin{abstract}
Coarctation of the Aorta ( $\mathrm{CoA}$ ) accounts for approximately $5 \%$ of all congenital heart defects worldwide, with a three-fold greater prevalence in males than females.1 Coractation patients usually present at a young age. Studies show that the median age of referral to a pediatric cardiologist is 5.8years, with the mean age being 8.4years. 2 The novelty of this case lies in the fact that the patient was in his mid-30s. Coarctation of the Aorta in an adult is a rare phenomenon. The pathology demands treatment by either surgical repair or catheter intervention. Over the past decade, endovascular repair has fast gained popularity. Most of the case reports and studies regarding endovascular repair of $\mathrm{CoA}$ are found in the paediatric cardiology literature. As demonstrated in this case, successful treatment of a complex disease was achieved by Interventional Radiology using minimally invasive endovascular techniques.
\end{abstract}

Keywords: coarctation of aorta, congenital, endovascular repair
Volume 3 Issue 6 - 2017

Zakariya Irfanullah
Ziauddin University, Pakistan

Correspondence: Zakariya Irfanullah, Ziauddin University, Khe-e-Shaheen, Phase 8, DHA, Karachi, Pakistan, Tel 3362292625, Email zirfanullah@gmail.com

Received: July 04, 2017 | Published: August II, 2017

\section{Key messages}

Coarctation of the Aorta in an adult is a rare phenomenon. The pathology demands treatment by either surgical repair or catheter intervention. Over the past decade, endovascular damage has fast gained popularity. In this report, we recount a successful treatment of a rare condition using minimally invasive, endovascular techniques.

\section{Introduction}

Coarctation of the Aorta (CoA) accounts for approximately 5\% of all congenital heart defects worldwide, with a three-fold greater prevalence in males than females. ${ }^{1}$ Because it frequently occours side by side with other abnormalities such as bicuspid aortic valve and ventricular septal defect, it is often missed out in the differential. This makes it all the more necessary to use radiological methods such as MRI and CT scanning to diagnose it correctly. Most of the case reports and studies regarding endovascular repair of $\mathrm{CoA}$ are found in the paediatric cardiology literature. As demonstrated in this case, successful treatment of a complex disease was achieved by Interventional Radiology using minimally invasive endovascular techniques.

\section{Case history}

A 35year-old male patient was referred to our interventional radiology clinic by a cardiothoracic surgeon with a diagnosis of possible coarctation of the aorta with concurrent severe aortic stenosis. His primary complaint was severe shortness of breath and lower extremity weakness with minimal exertion. His symptoms had been progressively worsening and at presentation he could barely walk a distance of 15 meters before stopping to rest. On physical examination, he had bounding bilateral radial pulses with faintly palpable femoral pulses. A CT angiogram of the chest was ordered which demonstrated a severe focal stenosis of the descending thoracic aorta, $2 \mathrm{~cm}$ distal to the takeoff of the left subclavian artery. The aorta measured $18 \mathrm{~mm}$ proximal to the stenosis and $20 \mathrm{~mm}$ distally. The proximal left subclavian artery measured $18 \mathrm{~mm}$. Normal great vessel branching anatomy was noted. The distal thoracic/abdominal aorta, as well as the iliac and femoral vessels were widely patent with no evidence for atherosclerotic disease. Left ventricular hypertrophy was also noted on the CT scan. Given the history of severe aortic stenosis an echocardiogram was performed which demonstrated mild concentric left ventricular hypertrophy. A thickened and calcified aortic valve was noted with severe aortic stenosis and an aortic valve peak gradient of $100 \mathrm{mmHg}$. The estimated pulmonary artery systolic pressure was $73 \mathrm{mmHg}$. Given the constellation of findings on the chest CTA, and after consultation with the referring thoracic surgeon, a decision was made to proceed with catheter angiography with a plan to treat via an endovascular approach. The surgeon would then repair the aortic valve once the coarctation had been treated.

The patient was brought to the endovascular suite and the right groin was prepped. Moderate conscious sedation was administered during the case via a peripheral IV. Access was gained into the right common femoral artery and a 6 French sheath was placed. A pigtail catheter was then placed in the mid descending thoracic aorta and an angiogram was performed demonstrating severe narrowing of the thoracic aorta. The left subclavian artery was not visualized, multiple collateral vessels were seen arising from the mid thoracic aorta. Initial attempts at crossing the stenotic segment with a glide wire and cobra catheter were unsuccessful. The left arm was then prepped and access was gained into the left brachial artery and a 5 Fr sheath was placed. A pigtail catheter was then advanced into the aortic arch and an arch aortogram was performed demonstrating normal branching anatomy with delayed filling of the distal thoracic aorta noted. Simultaneous pressure measurements proximal and distal to the stenosis demonstrated a gradient of $40 \mathrm{mmHg}$. After repeated attempts the stenosis was finally crossed from below using a glide wire and cobra catheter. The glide wire was then exchanged for a $260 \mathrm{~cm}$ Double Curved Lunderquist wire (Cook Medical). The stenotic segment was predilated with an $8 \mathrm{~mm}$ by $4 \mathrm{~cm}$ balloon with resolution of the tight waist seen at $10 \mathrm{~atm}$. The 6 French right groin sheath was then upsized to a $11 \mathrm{Fr}$ sheath. A $20 \mathrm{~mm}$ diameter by $55 \mathrm{~mm}$ long Wallstent (Boston Scientific) was then placed across the stenotic segment with the proximal end of the stent positioned just at the origin of the left subclavian artery and the distal end within the descending 
thoracic aorta past the focal stenotic area. As the stent was unsheathed it migrated distally and opened up distal to the stenotic segment. A $3.9 \mathrm{~cm}$ long covered balloon expandable Cheatham-platinum (CP) stent (NuMed Inc) was then selected and hand crimped on a $16 \mathrm{~mm}$ by $6 \mathrm{~cm}$ long Z-MED (NuMed Inc) balloon. The existing short $11 \mathrm{Fr}$ sheath was replaced for a $70 \mathrm{~cm}$ long $11 \mathrm{Fr}$ Mullens sheath which was advanced past the stenotic segment. The balloon mounted stent was then accurately positioned once again, taking care not to cover the left subclavian artery. The sheath was pulled back repeat angiogram performed from the left subclavian artery catheter, and the balloon was carefully inflated. The balloon was deflated and removed. Completion angiogram from the arm approach demonstrated accurate placement of the stent with significant improvement in luminal diameter noted. Repeat simultaneous pressure measurements across the stent demonstrated no residual gradient. The patient was then transferred to the recovery bay were the sheath was removed once the ACT was below 170 . The patient had an uneventful post-op recovery and was discharged the following day. At one week clinical follow up, the patient reported significant improvement in symptoms (Figures 1-4).

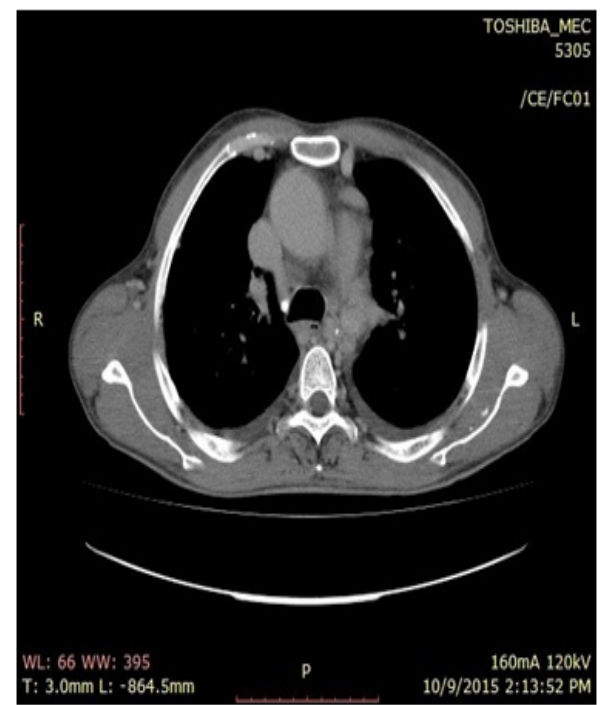

Figure I CT Angiogram demonstrating a severe focal stenosis of the descending thoracic aorta $2 \mathrm{~cm}$ distal to the takeoff of the left subclavian artery.

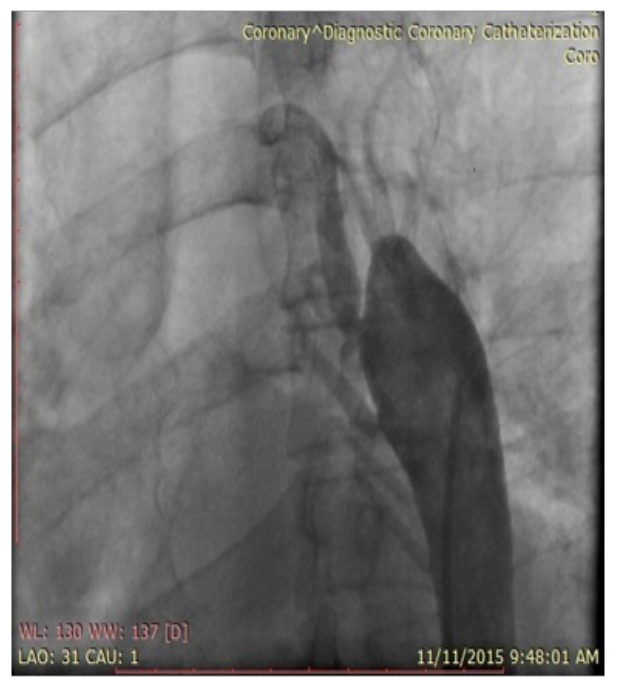

Figure 2 Aortogram from the ascending aorta (left brachial approach) demonstrated severe occlusion of the thoracic aorta just pass the origin of the left subclavian artery with multiple thoracic collaterals noted.

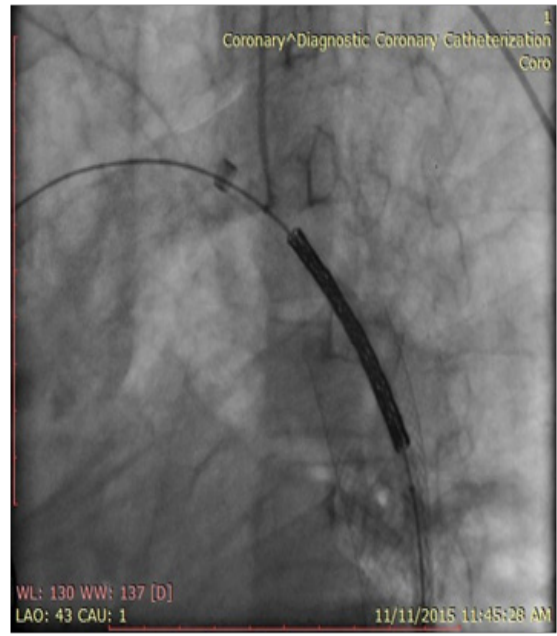

Figure 3 Stent positioned across stenotic area and successfully deployed.

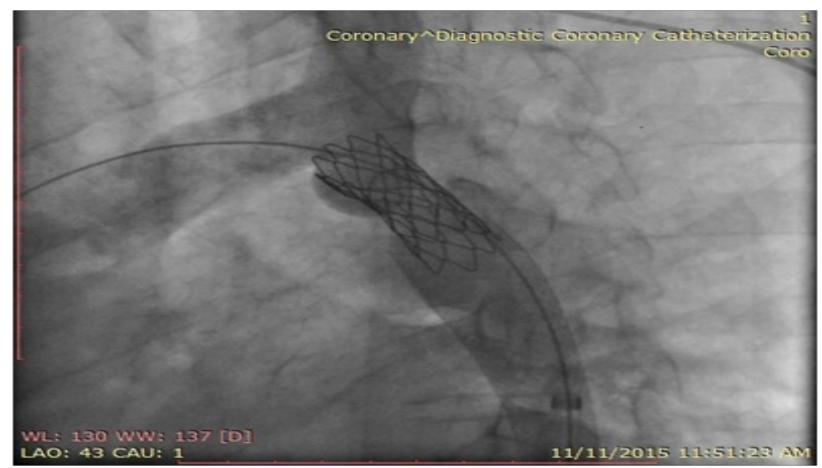

Figure 4 Completion angiogram demonstrated accurate placement of the stent with significant improvement in the luminal diameter.

\section{Discussion}

Coractation patients usually present at a young age. Studies show that the median age of referral to a pediatric cardiologist is 5.8 years, with the mean age being 8.4 years. ${ }^{2}$ The novelty of this case lies in the fact that the patient was in his mid-30s. He presented with severe shortness of breath on mild exertion and disparity in the pulses of his upper and lower extremities.

CoA is a narrowing of the aorta, usually beyond the blood vessels that branch off to the upper body and before the blood vessels that lead to the lower body. Classically, a constriction in the thoracic aorta distal to the origin of the left subclavian artery is seen, at approximately the level of the ductal structure. A common finding in CoA patients is the formation of collateral vessels that connect arteries from the upper part of the body to the vessels below the level of coarctation. ${ }^{3}$

A study conducted by the BMJ evaluated the hypothesis that endovascular management of $\mathrm{CoA}$ is more effective than balloon angioplasty, and has less late complications and side effects, including, but not limited to aneurysms, acute dissection and restenosis. The study confirmed the hypothesis but went on to mention that approximately one-fifth of the patients who underwent angiography came back with aortic dilatation. ${ }^{4}$

\section{Conclusion}

Coarctation of the Aorta in an adult is a rare phenomenon. The pathology demands treatment by either surgical repair or catheter 
intervention. Over the past decade, endovascular damage has fast gained popularity.

\section{Acknowledgements}

None.

\section{Conflict of interest}

Author declares that there is no conflict of interest.

\section{References}

1. Jenkins $\mathrm{N}$, Ward C. Coarctation of the aorta: natural history and outcome after surgical treatment. QJM. 1999;92(7):365-371.
2. Strafford MA, Griffiths SP, Gersony WM. Coarctation of the aorta: a study in delayed detection. Pediatrics. 1982;69(2):159-163.

3. Rao PS. Coarctation of the aorta. Current cardiology reports. 2005;7(6):425-434.

4. Harrison D, McLaughlin P, Lazzam C, et al. Endovascular stents in the management of coarctation of the aorta in the adolescent and adult: one year follow up. Heart. 2001;85(5):561-566. 